\title{
Smart Home Application based on CloudDB
}

\author{
Nan Ding, Fangqin Xu \\ Department of Computer Science and Technology, Shanghai JianQiao University, Shanghai, 201319, China \\ dingnan_david@126.com, xufqin2009@126.com
}

\begin{abstract}
While the IOT, Smart Home ZigBee Protocol and CloudDB tech is still in developing in China area, this paper introduces a Smart Home Manage System designed by Visual Studio 2010, a system uses ZigBee Protocol to transmit data and share the database $24 / 7$ by CloudDB, a system promoting the comfortability of an apartment with auto control, manage the furniture and auto detect the big environment.

Index Terms - IOT, ZigBee, Smart Home, CloudDB
\end{abstract}

\section{Environment Introduction}

A. IOT

The full name of IOT is "The Internet of Things", which is a network connecting everything to the internet for them to exchange information and communicating according to the appointed protocol to realize intelligent identification, position locating, tracing, monitoring and managing by sorts of information sensors as RFID, infrared sensor, Global Position System (GPS), laser scanner and Gas Inductor,

The IOT was called "The third wave of world information industry development" behind the Computer and Network because of the widely use of intelligent sensing, identification technique and pervasive calculating in the merging of the network. IOT is extended internet, it is business and application more than a network, it included the internet and all of its resources, it's compatible with all of the application in the internet whereas each one of the elements of IOT is personal and privatization.

After decades of development, IOT is now comprehensively used in Intelligent Transportation, environmental protection, public security, intellectual fire-fighting, industry monitoring, etc. [1]. The detailed application contains Cattle Traceability, Personal Health, Secure City Smart Traffic and so on [2].

\section{B. ZigBee}

ZigBee is a wireless personal corresponding protocol based on IEEE802.15.4 standard, characterized by its low complexity, short range, low-power dissipation, self-organization, low speed and low cost [3]. ZigBee is splendid on deploying large-scale networking, as a result, all the nodes can join and rejoin the network frequently.

The bottom layer of Zigbee concluded Media Access Layer and Physical Layer standard by IEEE 802.15.4. IEEE 802.15.4 standard named two different physical spectrum. The higher spectrum can spread in the air in the $250 \mathrm{~km} / \mathrm{s}$, the lower one spread in $20 \sim 40 \mathrm{~km} / \mathrm{s}$. Because of the package consumption and delay, practical data throughout would be lower than the standard Bit rate.

\section{Smart Home}

Smart Home assembles every single artificial and automatical electricity device related to the daily life through progressive computer, network, artificial, wireless and control tech. Smart Home proposes to facilitate and modernize the house. With IOT rises, current Smart Home is mainly about Smart Home based on IOT [4].

\section{CloudDB}

CloudDB is a cloud computing tech developed with the SaaS (software-as-a-service) becomes the application trend, it strongly intensified the memory ability of database, took away the repeating redundant setting of people, software and hardware, made the updating of software and hardware easier, and virtualized a lot of back-end functions. CloudDB is featured by its high extension, high availability, using multi-rented form and supporting delivering resources efficiently. As it were, CloudDB is the future of database [5].

Not like the traditional database, the CloudDB is dynamically extensible. Theoretically, the CloudDB can be extended infinitely to satisfy the increasing data memory needs. The ClouldDB would be excellently flexible in the continuous volatile condition. For example, for an e-commerce company in product retail, there will be some seasonally or accidentally change in product demand. At this special moment, the additional data memory resources can be allocated to dealing with the rising requirement. This period would only be lasting a few minutes, once the requirement passed, all resources would be released instantly. High availability is also one advantage of CloudDB. CloudDB don't have the problem named SPF (Single Point of Failure). If a point of CloudDB happens to fail, the rest of the points would take over its unfinished business. Moreover, in a CloudDB, the data are always copied. All of the big cloud computing providers, such as Google, Amazon, IBM and etc., deploy data center all over the world and providing the high-quality fault-tolerant capability through copy data among different zones.

\section{Design of the Smart Home Application based on CloudDB}

\section{A. Basal Function of the system}

Such as Fig.1 described, system consists of three parts as Secure, Environment and Furniture Control. Each part uses ZigBee Routers for transmitting the information to the computer through the network created by the ZigBee Coordinator, and then manage and detecting the information in the operating surface wrote by $\mathrm{C \#}$. 


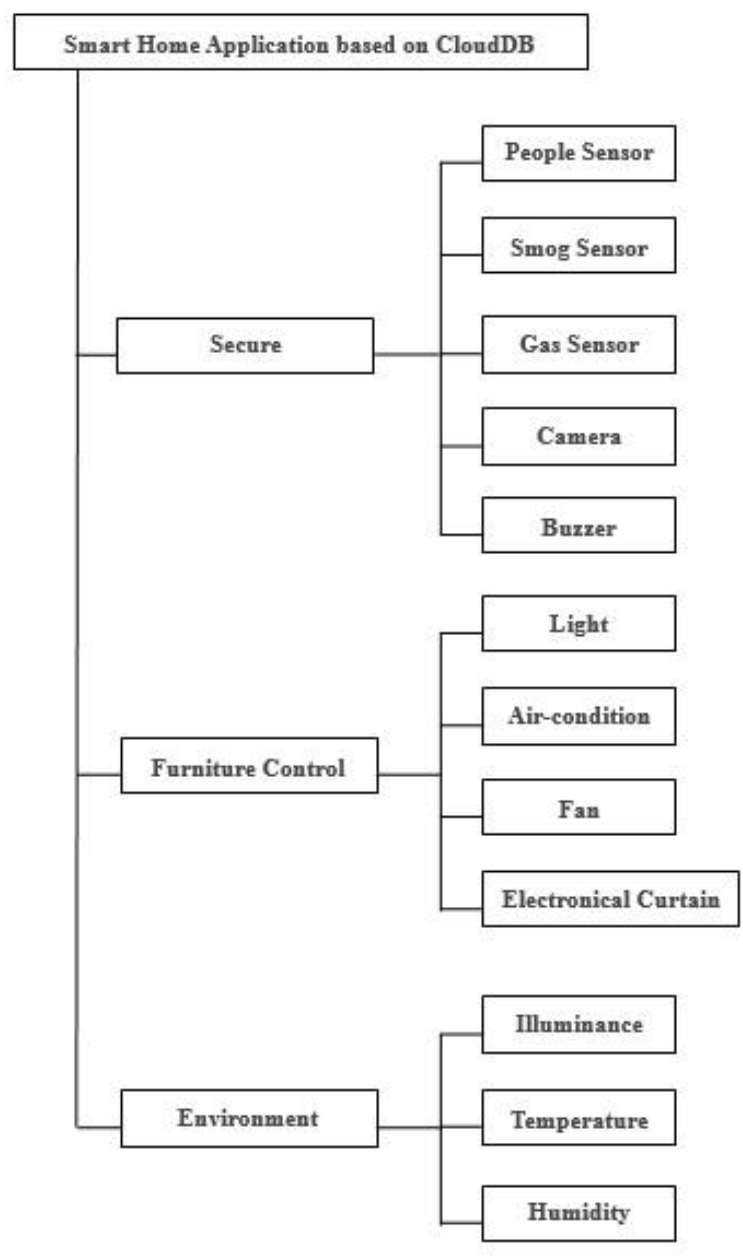

Fig.1.Function structure

\section{1) Secure}

Secure functioned with People sensor, Gas sensor, Smog sensor, Camera and Buzzer.

People sensors and Camera are used to protect the apartment from outside. Users can put the people sensors in some places could be broken in, like doors and windows. Once someone opens the windows or the doors, people sensors would send the message to system through ZigBee Routers at the very first time. The people sensors can be aided with Camera Function. There always be some misinformation sent, users can avoid the situation by opening the Cameras, which can be settled in the living room or balcony, to check the house visually, ensure the safety without wasting the user's time.

The threat from the apartment itself do no less harm than the outsiders sometimes, fire disaster and gas leak could make loses on the users' property, or even danger to their own life. Thus, Secure aided with gas and smog detection on purpose to avoid fire those two. Users can place the sensors, which detect concentrations of $\mathrm{CO}$ and $\mathrm{CO}_{2}$ in the air every few seconds, in wherever might catch fire, kitchen area mostly. The smokers could also put a smog sensor in their smoking area of the apartment.

\section{2) Furniture Control}

Furniture Control concludes controlling lights, air-conditioners, fans and electrical curtains. The fans are enabled by the DC motor while Electronical curtains are enabled by the stepping motors, which rotating clockwise to open the curtains, rotating counterclockwise to close. The electrical lights and lamps in the house can be operated individually. All of the furniture can be controlled by the system without any remote controller.

Furniture Control set a buzzer for warning people living around. People can turn the buzzer on in emergencies, such as disables can't leave the house when there's a fire disaster. When the buzzer's turned on, the neighbours would notice the loud noise and call 119.

\section{3) Environment}

Environment constitutes by illuminance detection, humidity detection and temperature detection, which detected by humidity, illuminance and illuminance sensors. These three kinds of sensors can collect environment information of the house in a regular period, and send to the system by ZigBee Routers and the ZigBee Coordinator. The system would analyze the information and present it to the users as exact numbers.

\section{B. Structure of the system}

As Fig.2 described, the structure of the Smart Home Application based on CloudDB is including the Time Control, the Sensor Control, the Manual Control, the Administrator and CloudDB. All of the first three modes are for the ordinary users, the Administrator is used by the supervisors to manage the system. The CloudDB is the basal function of the system.

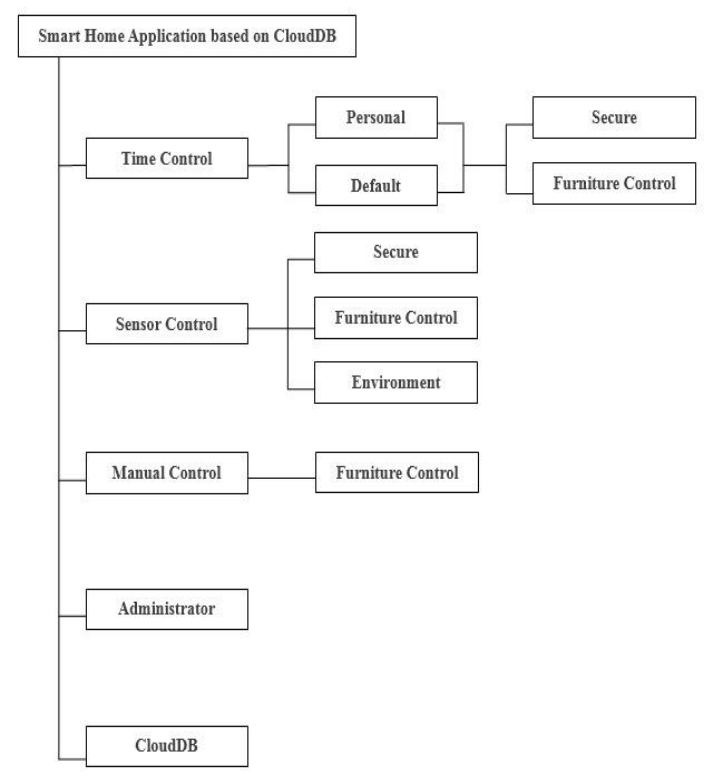

Fig.2.System Structure

\section{1) Time Control}

Time Control uses the Control function of the system and has two different modes, Personal and Default. In the Personal mode, users are able to set any furniture in the apartment on 
or off at any time of a day with their own favor. For example, users can let the system open the curtain at 6 a.m., close the curtain and open the light in the living room at 6 p.m. In the Time Control, a user can look up all his/her furniture setting conveniently, and also can easily insert or delete one.

Considered there might be some users don't have much personal favor or too lazy to set their Time Control, the system offered a default button, which has already set all the furniture in reasonable time.

Time Control combined Control and Secure, and made a series of secure setting acquiescently. For example, in the detecting area of concentrations of $\mathrm{CO}$ and $\mathrm{CO}_{2}$, the concentration status would be labeled as "Normal" or "High". Once there was a fire or gas leaking and the concentrations of $\mathrm{CO}$ or $\mathrm{CO}_{2}$ is higher than the normal condition, the label would change to "High" and the system would automatically turn the buzzer on to warn the users around. The buzzer would keep ringing till the concentration gets lower and the label come back to the "Normal".

\section{2) Sensor Control}

Sensor Control uses all of the three functions. Sensor Control would show the environment information of the apartment of the current time. The information contains the illuminance, humidity, temperature, concentrations of $\mathrm{CO}$ and $\mathrm{CO}_{2}$ of the house. The users can set the furniture changed by the environment information of their home. For example, as the temperature sensors detecting shows the temperature of the apartment is higher than 30 centigrade degrees, the air-conditioner would turn on automatically and set to 20 centigrade degrees, when the temperature of the apartment is lower than 25 centigrade degrees, the air-conditioner would turn off, or the lights would turn on while the illuminance is lower than 40Lux.

Considering the security, Sensor Control also contains the acquiescent secure setting mentioned in Time Control to dealing with the unpredictable accident.

\section{3) Manual Control}

The system deployed the Manual Control as a Contingency Plan, in case Time Control and Sensor Control disabled. The function of the Manual is simply controlling furniture. If the furniture of the apartment can't work as the set configuration, like the buzzer keeping beeping in the normal situation, the users can open the Manual Control and close the buzzer directly. When the Manual Control is on, the system would ignore all of the settings of Time Control and Sensor Control.

4) Administrator

In the Administrator, the supervisor is authorized to look up the information of every user, including the time of a user login and logout, his/her setting of Time Control and Sensor Control, the record of Manual Control, including the operated furniture and the operating time. The supervisor can change the authority of users. A user canceled authority would not be able to login the system until the supervisor recovered his/her authority. A new register would have to be authorized by the supervisor before he/she can login.

\section{5) $C l o u d D B$}

The system takes SQL Azure as the database, the data would be uploaded to the server and shared by all of the terminals installed the system. Thus, wherever the users goes, they can change a temporary apartment to their own house after they login the Smart Home Application. The Smart Home Application based on CloudDB is suitable for Multiple Hotels especially.

\section{Tech}

The system was developed by Visual Studio 2010, which support the object-oriented programming mechanism. Widgets being deployed to accomplish the surface of the system, then code wrote with $\mathrm{C \#}$ to collect data from sensors, control furniture, connecting database, and eventually, the management and supervision of the Smart Home was truly realized. Microsoft SQL Azure was used to create the database, memorize and share the data with all terminals installed the system.

\section{Conclusions}

Analyzed the environment of IOT, ZigBee protocol, Smart Home, the system realized an expedient and functional, comprehensive and practical Smart Home Application. Furthermore, the system was combined with the CloudDB tech in fleetly developing, which extended the system's availability from home to multiple hotels, from personal apartments to the public areas. Benefits from the effort of so many intelligent people made on Smart Home, the system would also benefits to the spread of Smart Home.

\section{References}

[1] Yuan Yuan. Status and Development Trend of the Internet. Computer CD Software and Application. 2011, 5: 117.

[2] HE Dong. The Application and Development of the Technology of the Internet of Things. SCI-Tech Information Development \& Economy. 2012, 22(8): 86.

[3] WANG Bo. Smart Home System Internal Network Based on Zigbee Technology. Electronic Sci. \& Tech. 2012, 25(8): 114.

[4] Zi Xuan. 2012 Smart Home Consumer treads. Radio Frequency World. 2012, 1: 42-43.

[5] Lin ZY, Lai YX, Lin C, Xie Y, Zou Q. Research on cloud databases. Journal of Software. 2012, 23(5): 1148. 